QUARTERLY OF APPLIED MATHEMATICS

VOLUME LXIX, NUMBER 4

DECEMBER 2011, PAGES 759-770

S 0033-569X(2011)01238-X

Article electronically published on July 1, 2011

\title{
GLOBAL SOLVABILITY FOR THE HEAT EQUATION WITH BOUNDARY FLUX GOVERNED BY NONLINEAR MEMORY
}

\author{
BY \\ JEFFREY R. ANDERSON (College of Science, Technology, Engineering and Mathematics, \\ University of Wisconsin-Stout, Menomonie, Wisconsin 54751), \\ KENG DENG (Department of Mathematics, University of Louisiana at Lafayette, Lafayette, \\ Louisiana 70504), \\ AND
}

ZHIHUA DONG (Department of Mathematics, University of Louisiana at Lafayette, Lafayette, Louisiana 70504)

Abstract. We introduce the study of global existence and blowup in finite time for the heat equation with flux at the boundary governed by a nonlinear memory term. Via a simple transformation, the model may be written in a form which has been introduced in previous studies of tumor-induced angiogenesis. The present study is also in the spirit of extending work on models of the heat equation with local, nonlocal, and delay nonlinearities present in the boundary flux. Additionally, we provide a brief summary of related studies regarding heat equation models where memory terms are incorporated within reaction or diffusion.

1. Introduction. We investigate the global solvability and blowup in finite time for the heat equation with flux at the boundary governed by a nonlinear memory law

$$
\begin{array}{ll}
u_{t}=\Delta u & \text { on } \Omega_{T}, \\
\nabla u \cdot \mathbf{n}=u^{q} \int_{0}^{t} u^{p}(\cdot, s) d s & \text { on }(\partial \Omega)_{T}, \\
u=u_{0} & \text { on } \bar{\Omega} \times\{0\} .
\end{array}
$$

Here, $p \geq 0, q \geq 0$, and $\Omega_{T}=\Omega \times(0, T)$, where $\Omega$ is a bounded domain in $\mathbf{R}^{N}$ having piecewise smooth boundary $\partial \Omega$ with outward pointing unit normal $\mathbf{n}$. The initial condition $u_{0}$ is a nonnegative, continuous function on $\bar{\Omega}$. Our primary result is that if

Received April 28, 2010.

2010 Mathematics Subject Classification. Primary 35B44, 35K05, 35K20.

Key words and phrases. Global existence, finite time blowup, memory boundary condition.

E-mail address: andersonjeff@uwstout.edu

E-mail address: deng@louisiana.edu

E-mail address: zxd5200@louisiana.edu

(C)2011 Brown University Reverts to public domain 28 years from publication 
$0 \leq p+q \leq 1$, then every solution of (1) is global. On the other hand, if $p+q>1$, then all nontrivial, nonnegative solutions blow up in finite time.

There are two main sources of motivation for the present study of (1). First, we conduct our investigation in parallel to previous studies of the semilinear heat equation model with memory,

$$
\begin{array}{ll}
u_{t}=\Delta u+u^{q} \int_{0}^{t} u^{p}(\cdot, s) d s & \text { on } \Omega_{T}, \\
u=0 & \text { on }(\partial \Omega)_{T}, \\
u=u_{0} & \text { on } \bar{\Omega} \times\{0\},
\end{array}
$$

and the localized boundary flux version of (1),

$$
\begin{array}{ll}
u_{t}=\Delta u & \text { on } \Omega_{T}, \\
\nabla u \cdot \mathbf{n}=u^{q+p} & \text { on }(\partial \Omega)_{T}, \\
u=u_{0} & \text { on } \bar{\Omega} \times\{0\} .
\end{array}
$$

It is known that all solutions of (2) are global in the case $0 \leq p+q \leq 1$ [20, 29, 35, 37, and the same result is also true for solutions of (3); e.g., see [26]. On the other hand, while all nontrivial solutions of (3) blow up in finite time if $p+q>1$ [28, 34, the situation is slightly different for the model with memory terms in the reaction. In the case $p+q>1$ with $q<1$, all nontrivial solutions of (2) blow up in finite time, while in the case $p+q>1, q \geq 1$, solutions of (2) which blow up in finite time and which are global both exist depending upon the size of $u_{0}$ [20, 29].

Our second source of motivation for the study of (1) originates with a model of capillary growth in solid tumors as initiated by angiogenic growth factors [27. In this reference a model for the transmission of growth factors across a capillary wall is introduced in the following form:

$$
\begin{array}{ll}
u_{t}=\nabla \cdot(\nabla \phi(x, t, u)+\mathbf{f}(x, t, u))+h(x, t, u) & \text { on } \Omega_{T}, \\
(\nabla \phi(x, t, u)+\mathbf{f}(x, t, u)) \cdot \mathbf{n}=g(x, t, u, v) & \text { on }(\partial \Omega)_{T}, \\
u=u_{0} & \text { on } \bar{\Omega} \times\{0\}
\end{array}
$$

with $g_{v} \geq 0$ on $(\partial \Omega)_{T} ; g_{v} \equiv 0$ on $(\partial \Omega \backslash \Sigma)_{T}$, and

$$
\begin{array}{ll}
v_{t}=F(x, t, u, v)+G(u)_{t} & \text { on } \Sigma_{T}, \\
v=v_{0} & \text { on } \Sigma \times\{0\} .
\end{array}
$$

Here, $\Sigma$ is a relatively open subset of $\partial \Omega$, which represents the capillary wall.

In (1), if we let

$$
v(x, t) \equiv \int_{0}^{t} u^{p}(x, s) d s
$$

and $\Sigma=\partial \Omega$, then the model may be seen to be of the type (4)-(5), with $g=u^{q} v$, $F=u^{p}, G=0$, and $v_{0}=0$. In previous works, we have studied existence, uniqueness, and blowup in finite time for various forms of (4)-(5) [2, 3. However, blowup results in these references are only those which may be achieved through comparison with a related localized model. With the present study, we intend to provide a more complete analysis, 
albeit for a specialized version of (4)-(5), via identification with a heat equation problem involving memory flux at the boundary (1).

Considering the nonlinear time integral condition governing flux through the boundary, the model (1) involves a continuous time delay, often referred to as an integrodifferential system or a memory condition in the literature. Models involving memory terms in reaction have arisen in studies of nuclear reactor dynamics [24, 32] and population dynamics 25], specifically in the case of logistic growth models involving both nondelayed and hereditary effects [7, 40. Local/global existence, stabilization, and blowup in finite time of solutions for a variety of generalizations of such models have subsequently been investigated within a number of previous works, e.g., [6, 12, 36, 38, 39.

Memory terms in diffusion have been studied as well, arising in models of viscoelastic forces in non-Newtonian fluids [18, 31] and resulting from a modified Fourier law applied to an anisotropic, nonhomogeneous media [41]. Numerical studies of closely related models and also those involving a form of the Fisher equation with memory may be found in [4, 5, 8. It is useful to note here that fractional order time derivatives, as memory operators, have been studied in a memory formalism of D'Arcy's law and molecule transport across a biological membrane. See [9, 10, and the references therein. Memory terms in diffusion and reaction have additionally been introduced in the context of climate models [15, 19.

Despite the volume of work done on models incorporating memory in reaction, diffusion, or both, there appear to be very few appearances in the literature of diffusion models in which such terms are present in the boundary flux. In [11, a linear memory term of the type in (1), with $q=0, p=1$, is introduced as arising in hereditary boundary conditions of Newtonian radiation and calorimetry. Similar hereditary boundary conditions have been employed in models of time-dependent electromagnetic fields at dissipative boundaries [16]. Boundary conditions incorporating convolution in time have been studied more extensively in the case of wave equation models arising in shock problems involving a linear viscoelastic bar; see, e.g., 30, 33] and the references therein. We have been able to locate only one general study of existence of weak solutions for a quasilinear parabolic system in which memory terms are incorporated in diffusion, reaction, and boundary flux conditions [1]. Owing to our need for comparison results for classical solutions, we have developed these fundamental results for (1) herein.

The plan of the paper is as follows. In Section 2 we present the local existence and the comparison principle. In Section 3 we prove that for $p+q \leq 1$, every solution of (1) is global, while for $p+q>1$, all solutions blow up in finite time. In Section 4 we show that for $p>1$ or $q>1$, blowup can occur only on the boundary.

2. Local existence and comparison results. In this section, we establish the existence of classical solutions, along with sub- and super-solution comparisons for (1). While these arguments are fairly standard, we provide them here as a way to introduce the Green's function formulation, to be utilized later, and to present comparison results as applicable even in the non-Lipschitz cases $p, q<1$. For such purposes, we begin by defining $G_{N}(x, y, t, \tau)$ as the Green's function for the heat equation with homogeneous Neumann boundary condition. $u(x, t) \in C^{2,1}\left(\Omega_{T}\right) \cap C\left(\overline{\Omega_{T}}\right)$ is a classical solution of (1); 
i.e., $u$ is $C^{1}$ in $t, C^{2}$ in $x$ on $\bar{\Omega} \times(0, T)$ and continuous on $\bar{\Omega} \times[0, T]$ if and only if

$$
\begin{aligned}
u(x, t)= & \int_{\Omega} G_{N}(x, y, t, 0) u_{0}(y) d y \\
& +\int_{0}^{t} \int_{\partial \Omega} G_{N}(x, y, t, \tau) u^{q}(y, \tau) \int_{0}^{\tau} u^{p}(y, s) d s d S_{y} d \tau
\end{aligned}
$$

for all $x \in \bar{\Omega}, 0 \leq t \leq T$.

We prove local existence of solutions of (1) via a fixed point argument. Despite the presence of a memory term, the necessary steps are nearly identical to well-known results for corresponding localized problems [17. Thus, we do not repeat all of the details here. Although presented in the context of (1), the work below may be applied to more general uniformly parabolic models and nonlinear memory terms. We restrict this discussion to nonnegative solutions; however, such a condition may also be removed.

Given $u \in C(\bar{\Omega} \times[0, T])$, define

$$
\begin{aligned}
\mathcal{T}[u](x, t) \equiv & \int_{\Omega} G_{N}(x, y, t, 0) u_{0}(y) d y \\
& +\int_{0}^{t} \int_{\partial \Omega} G_{N}(x, y, t, \tau) u^{q}(y, \tau) \int_{0}^{\tau} u^{p}(y, s) d s d S_{y} d \tau
\end{aligned}
$$

and

$$
M_{0} \equiv \sup _{\bar{\Omega}}\left|u_{0}\right| .
$$

Continuing in a manner also similar to [13, let

$$
\kappa(t) \equiv \sup _{x \in \bar{\Omega}, 0 \leq \tau \leq t} \int_{0}^{\tau} \int_{\partial \Omega} G_{N}(x, y, \tau, \eta) d S_{y} d \eta .
$$

We note that $\kappa(t) \leq 2 C_{0} \sqrt{t}$ for constants $\varepsilon_{0}, C_{0}>0$ with $t<\varepsilon_{0}$ 22. Fix $\hat{T}<\varepsilon_{0}$ and $M>M_{0}$ so that

$$
M^{q+p} \hat{T} \kappa(\hat{T}) \leq M-M_{0}
$$

If $|u| \leq M$ and $0 \leq t \leq \hat{T}$, then

$$
\begin{aligned}
|\mathcal{T}[u]| & \leq M_{0} \int_{\Omega} G_{N}(x, y, t, 0) d y+M^{q+p} \hat{T} \kappa(\hat{T}) \\
& \leq M_{0}+\left(M-M_{0}\right) .
\end{aligned}
$$

So, $\mathcal{T}: K \rightarrow K$, where

$$
K \equiv\left\{u \in C(\bar{\Omega} \times[0, \hat{T}]):\|u\|_{\infty} \leq M\right\} .
$$

The continuity and compactness of $\mathcal{T}$ follow from the same arguments as in [17. Therefore, $\mathcal{T}$ has a fixed point, which is a solution of (1), and, by standard results, is a classical solution and may be continued unless $\|u\|_{\infty}$ becomes unbounded.

Uniqueness and comparison results for (1) may be developed using the same techniques as used in [3] for the transmission model $(4)-(5)$. Nevertheless, we herein provide an adaptation of this more general work to the case of classical sub- and super-solutions, towards a more complete comparison theory than is currently known for the case of nonlinear diffusion models addressed in this reference. In such respect, our treatment 
here actually bears a great deal more similarity to that of [29] in the use of classical maximum principles and development of the comparison results.

Consider $w(x, t) \in C^{2,1}\left(\Omega_{T}\right) \cap C\left(\overline{\Omega_{T}}\right)$ such that

$$
\begin{array}{ll}
w_{t} \geq \Delta w & \text { on } \Omega_{T}, \\
\nabla w \cdot \mathbf{n} \geq a w+b \int_{0}^{t} c w(\cdot, s) d s & \text { on }(\partial \Omega)_{T}, \\
w \geq 0 & \text { on } \bar{\Omega} \times\{0\}
\end{array}
$$

with bounded continuous functions $a$ and $b, c \geq 0$. For a positive smooth function $\xi$, which satisfies $\nabla \xi \cdot \mathbf{n} \geq \alpha \xi$ on $\partial \Omega$, let

$$
w(x, t)=e^{\lambda t} \xi(x) W(x, t) .
$$

The positive constants $\alpha, \lambda$ are chosen to satisfy

$$
\alpha>1+T\|b\|_{\infty}\|c\|_{\infty}
$$

and

$$
\lambda>\frac{\Delta \xi}{\xi}
$$

on $\bar{\Omega}$.

Standard calculations show that

$$
W_{t} \geq \Delta W+\frac{2 \nabla \xi}{\xi} \cdot \nabla W+\left(\frac{\Delta \xi}{\xi}-\lambda\right) W
$$

in $\Omega_{T}$. Therefore, $W$ cannot assume a negative minimum anywhere within $\Omega \times(0, T]$. Similarly, if $W\left(x_{0}, t_{0}\right)=\min W<0$ for some $x_{0} \in \partial \Omega$, at the point $\left(x_{0}, t_{0}\right)$ it follows that

$$
\nabla W \cdot \mathbf{n} \geq-W\left(x_{0}, t_{0}\right)\left[\nabla \xi \cdot \mathbf{n}-\xi-e^{-\lambda t} \xi b \int_{0}^{t} c e^{\lambda s} d s\right]>0,
$$

which is a contradiction. Therefore, $W \geq 0$ on $\overline{\Omega_{T}}$, and hence $w \geq 0$ also.

Now, for a (classical) supersolution $\bar{u}$ and a subsolution $\underline{u}$ of (1), we have that $w \equiv \bar{u}-\underline{u}$ satisfies (7), where

$$
\begin{gathered}
{\left[\bar{u}^{q}(x, t)-\underline{u}^{q}(x, t)\right] \int_{0}^{t} \bar{u}^{p}(x, s) d s \equiv a(x, t)[\bar{u}(x, t)-\underline{u}(x, t)],} \\
b(x, t) \equiv \underline{u}^{q}(x, t),
\end{gathered}
$$

and

$$
\bar{u}^{p}(x, t)-\underline{u}^{p}(x, t) \equiv c(x, t)[\bar{u}(x, t)-\underline{u}(x, t)] .
$$

Provided $\bar{u} \geq \delta>0$ and $\underline{u} \geq 0, a, b, c$ will be bounded for all cases $p \geq 0, q \geq 0$. It follows that if $\bar{u}(\cdot, 0) \geq \underline{u}(\cdot, 0)$, then $\bar{u} \geq \underline{u}$.

Considering a solution $u_{\delta}$ of (1) with initial condition $u_{0}+\delta$, we see that the limit

$$
U(x, t) \equiv \lim _{\delta \rightarrow 0^{+}} u_{\delta}(x, t)
$$

exists and yields a maximal solution of (1). In the case $p, q \geq 1$, this is, in fact, the unique solution of the problem. Furthermore, if $\bar{u}$ is a supersolution and $\underline{u}$ is a subsolution of (1) such that $\bar{u} \geq \delta$ for some positive constant $\delta$ and $\underline{u} \geq 0$, then $\bar{u}(\cdot, 0) \geq u_{0} \geq \underline{u}(\cdot, 0)$ implies $\bar{u} \geq U \geq \underline{u}$ on $\overline{\Omega_{T}}$. 
3. Global existence and finite time blowup. In this section, we begin with the global existence for solutions of (1), that is, for any $T>0, u(x, t) \leq C(T)<\infty$ in $\overline{\Omega_{T}}$ with some positive constant $C=C(T)$.

TheOREM 3.1. If $p+q \leq 1$, then every solution of (1) is global.

Proof. We seek a global supersolution $\bar{u}$ of (1). From [14, there exists a function $\varphi(x) \in C^{2}(\bar{\Omega})$ satisfying

$$
0<\varphi(x) \leq 1 \quad \text { in } \Omega \quad \text { and } \quad \nabla \varphi \cdot \mathbf{n} \geq 1 \quad \text { on } \partial \Omega .
$$

Let $m_{1}=\max _{\bar{\Omega}}|\nabla \varphi|$ and $m_{2}=\max _{\bar{\Omega}}|\Delta \varphi|$. We define

$$
\bar{u}=M e^{\lambda t+\varphi},
$$

where

$$
\begin{aligned}
M & =\max \left\{\left\|u_{0}\right\|_{L^{\infty}(\Omega)}, 1\right\}, \\
\lambda & =\max \left\{m_{1}^{2}+m_{2}, 1 / p\right\} .
\end{aligned}
$$

Then $\bar{u}$ satisfies

$$
\begin{array}{ll}
\bar{u}_{t} \geq \Delta \bar{u} & \text { on } \Omega_{T}, \\
\nabla \bar{u} \cdot \mathbf{n} \geq \bar{u}^{q} \int_{0}^{t} \bar{u}^{p}(x, \tau) d \tau & \text { on }(\partial \Omega)_{T}, \\
\bar{u}(x, 0) \geq u_{0}(x) & \text { on } \bar{\Omega} \times\{0\} .
\end{array}
$$

Hence, $\bar{u}$ is a desired supersolution.

We then establish the blowup result. To this end, we first consider the following problem:

$$
\begin{array}{ll}
u_{t}=\Delta u & \text { on } \Omega_{T}, \\
\nabla u \cdot \mathbf{n}=c \int_{0}^{t} u^{\mu}(\cdot, s) d s & \text { on }(\partial \Omega)_{T}, \\
u=u_{0} & \text { on } \bar{\Omega} \times\{0\},
\end{array}
$$

where $c, \mu$ are positive constants. From now on, without causing any confusion, we may use $c_{i}, C_{i}$ or $\tilde{c}_{i}(i=0,1,2, \ldots)$ to denote various positive constants.

LEMma 3.2. For any $c>0$, if $\mu>1$, all nonnegative solutions of (9) blow up in finite time.

Proof. The solution $u$ of (9) satisfies

$$
\begin{aligned}
u(x, t)= & \int_{\Omega} G_{N}(x, y, t, 0) u_{0}(y) d y \\
& +c \int_{0}^{t} \int_{\partial \Omega} G_{N}(x, y, t, \tau) \int_{0}^{\tau} u^{\mu}(y, s) d s d S_{y} d \tau
\end{aligned}
$$

for all $x \in \bar{\Omega}, 0 \leq t \leq T$. As in 22 , one can show that

$$
\int_{\partial \Omega} G_{N}(x, y, t, \tau) d S_{x} \geq c_{0}>0 \quad \text { for } y \in \bar{\Omega}, T>t>\tau \geq 0 .
$$


By (10), (11) and Jensen's inequality, we have

$$
\begin{aligned}
\int_{\partial \Omega} u(x, t) d S_{x} & \geq c \int_{\partial \Omega}\left(\int_{0}^{t} \int_{\partial \Omega} G_{N}(x, y, t, \tau) \int_{0}^{\tau} u^{\mu}(y, s) d s d S_{y} d \tau\right) d S_{x} \\
& \geq c c_{0} \int_{0}^{t} \int_{0}^{\tau} \int_{\partial \Omega} u^{\mu}(y, s) d S_{y} d s d \tau \\
& \geq c_{1} \int_{0}^{t} \tau^{1-\mu}\left(\int_{0}^{\tau} \int_{\partial \Omega} u(y, s) d S_{y} d s\right)^{\mu} d \tau .
\end{aligned}
$$

On the other hand, by (10) and (11),

$$
\begin{aligned}
\int_{\partial \Omega} u(x, t) d S_{x} & \geq \int_{\partial \Omega} \int_{\Omega} G_{N}(x, y, t, 0) u_{0}(y) d y d S_{x} \\
& =\int_{\Omega} u_{0}(y) \int_{\partial \Omega} G_{N}(x, y, t, 0) d S_{x} d y \\
& \geq c_{0} \int_{\Omega} u_{0}(y) d y \\
& \geq c_{2}>0
\end{aligned}
$$

Set

$$
F(t)=\int_{0}^{t} \int_{\partial \Omega} u(x, \tau) d S_{x} d \tau \quad \text { for } t>0 .
$$

From (12) and (13), it follows that $F(t)$ satisfies

$$
F^{\prime}(t) \geq c_{4}+c_{3} \int_{0}^{t} \tau^{1-\mu} F^{\mu}(\tau) d \tau \quad \text { for } t>0
$$

Integration of the above inequality over $(0, t)$ then yields

$$
\begin{aligned}
F(t) & \geq c_{4} t+c_{3} \int_{0}^{t} \int_{0}^{\tau} \zeta^{1-\mu} F^{\mu}(\zeta) d \zeta d \tau \\
& \geq c_{4} t+c_{3} \int_{0}^{t}(t-\zeta) \zeta^{1-\mu} F^{\mu}(\zeta) d \zeta \\
& \geq c_{4} t+c_{3} t^{1-\mu} \int_{0}^{t}(t-\zeta) F^{\mu}(\zeta) d \zeta
\end{aligned}
$$

for $t>0$. Assume to the contrary that (9) has a global solution $u$. Then for any positive number $T$, we have

$$
F(t) \geq c_{4} T+c_{5} T^{1-\mu} \int_{T}^{t}(t-\zeta) F^{\mu}(\zeta) d \zeta \quad \text { for } T \leq t \leq 2 T .
$$

Thus, by comparison, $F(t) \geq H(t)$ on $[T, 2 T]$, where

$$
H(t)=c_{4} T+c_{5} T^{1-\mu} \int_{T}^{t}(t-\zeta) H^{\mu}(\zeta) d \zeta \quad \text { for } T \leq t \leq 2 T
$$

Clearly, $H(t)$ satisfies

$$
\begin{aligned}
& H^{\prime \prime}(t)=c_{5} T^{1-\mu} H^{\mu}(t), \quad T<t<2 T \\
& H(T)=c_{4} T \\
& H^{\prime}(T)=0
\end{aligned}
$$


Multiplying the equation in (16) by $H^{\prime}(t)$ and integrating from $T$ to $t$, we obtain

$$
H^{\prime}(t)=c_{6} T^{(1-\mu) / 2}\left(H^{\mu+1}(t)-H^{\mu+1}(T)\right)^{1 / 2} .
$$

Integration of this relation over $(T, 2 T)$ then leads to

$$
\begin{aligned}
c_{6} T^{(3-\mu) / 2}= & \int_{H(T)}^{H(2 T)}\left(z^{\mu+1}-H^{\mu+1}(T)\right)^{-1 / 2} d z \\
\leq & (\mu+1)^{-1 / 2} H^{-\mu / 2}(T) \int_{H(T)}^{2 H(T)}(z-H(T))^{-1 / 2} d z \\
& +2^{(\mu+1) / 2} \int_{2 H(T)}^{\infty} z^{-(\mu+1) / 2} d z \\
= & 2\left[(\mu+1)^{-1 / 2}+2(\mu-1)^{-1}\right] c_{4}^{(1-\mu) / 2} T^{(1-\mu) / 2},
\end{aligned}
$$

which is equivalent to

$$
T \leq 2\left[(\mu+1)^{-1 / 2}+2(\mu-1)^{-1}\right] c_{4}^{(1-\mu) / 2} / c_{6} .
$$

For sufficiently large $T$, inequality (17) yields a contradiction, which completes the proof.

We now consider the general case that $p+q>1$.

Theorem 3.3. If $p+q>1$, then all nonnegative solutions of (1) blow up in finite time.

Proof. We consider two cases.

Case $1(q<1)$. By the maximum principle, $u(x, t)>0$ for $x \in \bar{\Omega}$ and $t>0$. Let

$$
z=M u^{1-q},
$$

where $M=\left\|u_{0}\right\|_{L^{\infty}(\Omega)}^{q}$. Then $z$ satisfies

$$
\begin{array}{ll}
z_{t} \geq \Delta z & \text { on } \Omega_{T}, \\
\nabla z \cdot \mathbf{n}=M^{\frac{1-p-q}{1-q}}(1-q) \int_{0}^{t} z^{\frac{p}{1-q}}(x, \tau) d \tau & \text { on }(\partial \Omega)_{T}, \\
z(x, 0) \geq u_{0}(x) & \text { on } \bar{\Omega} \times\{0\} .
\end{array}
$$

$z$ is a supersolution of problem (9). Since $p /(1-q)>1$, by Lemma 3.2, $z$ blows up in finite time, and so does $u$.

Case $2(q \geq 1)$. Because the solution of the heat equation with homogenous Neumann boundary condition is a subsolution of (1), for a small $\sigma>0$, there exists a constant $c_{\sigma}>0$ such that the solution $u$ of (1) satisfies

$$
u(x, t) \geq c_{\sigma} \quad \text { for } x \in \partial \Omega, t \geq \sigma>0 .
$$

Let $q=\alpha+\gamma$, where $\gamma<1$ and $\gamma+p>1$. Then we have

$$
u^{q}=u^{\alpha+\gamma} \geq c_{\sigma}^{\alpha} u^{\gamma} \quad \text { for } x \in \partial \Omega, t \geq \sigma>0 .
$$


Consider the following problem:

$$
\begin{array}{ll}
\underline{u}_{t}=\Delta \underline{u} & \text { on } \Omega \times(\sigma, T), \\
\nabla \underline{u} \cdot \mathbf{n}=c_{\sigma}^{\alpha} \underline{u}^{\gamma} \int_{\sigma}^{t} \underline{u}^{p}(x, s) d s & \text { on } \partial \Omega \times(\sigma, T), \\
\underline{u}(x, \sigma) \leq u(x, \sigma) & \text { on } \bar{\Omega} .
\end{array}
$$

Proceeding analogously as in the proofs of Lemma 3.2 and case 1 of Theorem 3.3, we can show that $\underline{u}$ blows up in finite time. Since $u$ is a supersolution of (20), $u$ cannot exist globally.

4. Blowup on the boundary. In this section, we show that for problem (1) in the case $p>1$ or $q>1$, blowup cannot occur at the interior domain. For definiteness, we may assume that $T$ is the blowup time.

Theorem 4.1. If $p>1, q=0$ or $p \geq 0, q>1$, then blowup can occur only on the boundary.

Proof. We consider two cases.

Case $1(p>1, q=0)$. Following the general idea of [21], we set

$$
J(t)=\int_{0}^{t} \int_{0}^{\tau} \int_{\partial \Omega} u^{p}(y, s) d S_{y} d s d \tau .
$$

By (6), (11) and Jensen's inequality, we find

$$
J^{\prime \prime}(t) \geq c_{0}^{p}|\partial \Omega|^{1-p} J^{p}(t) .
$$

Multiplying both sides of the above inequality by $J^{\prime}(t)$ and integrating over $(0, t)$, we obtain

$$
J^{\prime}(t) \geq c_{7} J^{\frac{p+1}{2}}(t)
$$

which, upon integration over $(t, T)$, yields

$$
\int_{J(t)}^{\infty} s^{-\frac{p+1}{2}} d s \geq c_{8}(T-t)
$$

or equivalently,

$$
J(t) \leq c_{9}(T-t)^{-\frac{2}{p-1}} \quad \text { for } t \in[0, T) .
$$

We now take an arbitrary $\Omega^{\prime} \subset \subset \Omega$ with $\operatorname{dist}\left(\partial \Omega, \Omega^{\prime}\right)=\varepsilon>0$. For this $\Omega^{\prime}$, we then take $\Omega^{\prime \prime} \subset \subset \Omega$ such that $\Omega^{\prime} \subset \subset \Omega^{\prime \prime}, \operatorname{dist}\left(\partial \Omega^{\prime \prime}, \Omega^{\prime}\right) \geq \varepsilon / 3$ and $\operatorname{dist}\left(\partial \Omega, \Omega^{\prime \prime}\right) \geq \varepsilon / 3$. It is well known that for any $\varepsilon>0$,

$$
0 \leq G_{N}(x, y, t, \tau) \leq C_{\varepsilon} \text { for }|x-y| \geq \frac{\varepsilon}{3}, x, y \in \bar{\Omega}, 0<\tau<t<T,
$$

where $C_{\varepsilon}$ is a positive constant depending on $\varepsilon$. Then by (6), (21) and (22),

$$
\max _{\bar{\Omega}^{\prime \prime}} u(x, t) \leq C_{0}+C_{\varepsilon} J(t) \leq C_{1}(T-t)^{-\frac{2}{p-1}} .
$$

Proceeding similarly as in the proof of Theorem 4.1 of [23], we have

$$
u(x, t) \leq \frac{C_{3}}{\left[\psi(x)+\left(C_{2}+1\right)(T-t)\right]^{\frac{2}{p-1}}} \quad \text { in } \bar{\Omega}^{\prime} \times[0, T),
$$


where $\psi(x) \in C^{2}\left(\bar{\Omega}^{\prime}\right)$ satisfies

$$
\begin{gathered}
\psi(x)>0 \quad \text { in } \Omega^{\prime}, \quad \psi(x)=0 \quad \text { on } \partial \Omega^{\prime}, \\
\Delta \psi-\frac{p+1}{p-1} \frac{|\nabla \psi|^{2}}{\psi} \geq-C_{2} \quad \text { in } \Omega^{\prime}
\end{gathered}
$$

for $C_{2}>0$. Inequality (23) shows that $u(x, t)$ cannot blow up in $\Omega^{\prime} \times(0, T)$.

Case $2(p \geq 0, q>1)$. We now set

$$
\tilde{J}(t)=\int_{\sigma}^{t} \int_{\partial \Omega} u^{q}(y, \tau) \int_{\sigma}^{\tau} u^{p}(y, s) d s d S_{y} d \tau,
$$

where $0<\sigma<T / 4$. In view of Case 1 , it suffices to prove that $\tilde{J}(t)$ satisfies a similar estimate as (21). More precisely, if we can show that

$$
J(t) \leq \tilde{c}(T-t)^{-\beta} \quad \text { for } T / 2 \leq t<T,
$$

where $\tilde{c}, \beta>0$, then $u(x, t)$ must blow up on the boundary. By (19), we have that for $y \in \partial \Omega$ and $t \in[T / 2, T)$,

$$
\int_{\sigma}^{t} u^{p}(y, s) d s \geq c_{\sigma}^{p}(t-\sigma) \geq c_{\sigma}^{p} T / 4
$$

Let $\tilde{c}_{0}=c_{\sigma}^{p} T / 4$. Then

$$
\int_{\sigma}^{t} u^{p}(y, s) d s \geq \tilde{c}_{0} .
$$

On the other hand, by (11) we find

$$
\begin{aligned}
\int_{\partial \Omega} u(x, t) d S_{x} & \geq \int_{\partial \Omega}\left(\int_{0}^{t} \int_{\partial \Omega} G_{N}(x, y, t, \tau) u^{q}(y, \tau) \int_{0}^{\tau} u^{p}(y, s) d s d S_{y} d \tau\right) d S_{x} \\
& \geq c_{0} \int_{0}^{t} \int_{\partial \Omega} u^{q}(y, \tau) \int_{0}^{\tau} u^{p}(y, s) d s d S_{y} d \tau \\
& >c_{0} \int_{\sigma}^{t} \int_{\partial \Omega} u^{q}(y, \tau) \int_{\sigma}^{\tau} u^{p}(y, s) d s d S_{y} d \tau \\
& =c_{0} \tilde{J}(t) .
\end{aligned}
$$

Combining (25), (26) and applying Jensen's inequality, we further find that

$$
\begin{aligned}
\tilde{J}^{\prime}(t) & =\int_{\partial \Omega} u^{q}(y, t) \int_{\sigma}^{t} u^{p}(y, s) d s d S_{y} \\
& \geq \tilde{c}_{0} \int_{\partial \Omega} u^{q}(y, t) d S_{y} \\
& \geq \tilde{c}_{1}\left(\int_{\partial \Omega} u(y, t) d S_{y}\right)^{q} \\
& \geq \tilde{c}_{2} \tilde{J}^{q}(t) .
\end{aligned}
$$

Integration of the above inequality over $(t, T)$ yields

$$
\int_{\tilde{J}(t)}^{\infty} s^{-q} d s>\tilde{c}_{2}(T-t),
$$


which gives

$$
\tilde{J}(t) \leq \tilde{c}_{3}(T-t)^{-\frac{1}{q-1}} \quad \text { for } t \in[T / 2, T) .
$$

Hence, the proof is completed.

\section{REFERENCES}

[1] H. Amann, Quasilinear parabolic functional evolution equations. In Recent advances on elliptic and parabolic issues: Proceedings of the 2004 Swiss-Japanese Seminar: Zurich, Switzerland, 6-10 December, 2004, M. Chipot and H. Ninomiya, eds. World Scientific (2006), 19-44.

[2] J. R. Anderson, Long-time behavior of solutions of a nonlinear diffusion model with transmission boundary conditions, Dynam. Systems Appl. 18 (2009), 111-120. MR 2517554 (2010f:35168)

[3] J. R. Anderson, Unique solvability of a nonlinear diffusion model with transmission boundary conditions, Dyn. Contin. Discrete Impuls. Syst. Ser. A Math. Anal. 13B (2006), 159-170. MR2268788 (2007h:35156)

[4] A. Araujo, J. A. Ferreira, and P. Oliveira, The effect of memory terms in diffusion phenomena, J. Comput. Math. 24 (2006), 91-102. MR2196871(2006j:35127)

[5] A. Araujo, J. A. Ferreira, and P. Oliveira, Qualitative behavior of numerical traveling solutions for reaction-diffusion equations with memory, Appl. Anal. 84 (2005), 1231-1246. MR2178769 (2006k:65246)

[6] H. Bellout, Blow-up of solutions of parabolic equations with nonlinear memory, J. Differential Equations 70 (1987), 42-68. MR.904815 (89a:35032)

[7] A. Belmiloudi, Nonlinear optimal control problems of degenerate parabolic equations with logistic time-varying delays of convolution type, Nonlinear Anal. 63 (2005), 1126-1152. MR2211586 (2006m:49004)

[8] J. R. Cannon and Y. Lin, A priori $L^{2}$ error estimates for finite-element methods for nonlinear diffusion equations with memory, SIAM J. Numer. Anal. 27 (1990), 595-607. MR.1041253 (91b:65118)

[9] M. Caputo, C. Cametti, and V. Ruggero, Time and spatial concentration profile inside a membrane by means of a memory formalism, Physica A 387 (2008), 2010-2018.

[10] M. Caputo, 3-dimensional physically consistent diffusion in anisotropic media with memory, Rendiconti Lincei. Serie IX. Matematica e Applicazioni 9 (1998), 131-143. MR.1677270 (99k:76135)

[11] M. Ciarletta, A differential problem for heat equation with a boundary condition with memory, Appl. Math. Lett. 10 (1997), 95-101. MR1429481 (97j:80005)

[12] M. G. Crandall, S. O. Londen, and J. A. Nohel, An abstract nonlinear Volterra integrodifferential equation, J. Math. Anal. Appl. 64 (1978), 701-735. MR0500052 (58:17762)

[13] K. Deng, M. K. Kwong, and H. A. Levine, The influence of nonlocal nonlinearities on the long time behavior of solutions of Burgers' equation, Quart. Appl. Math. 50 (1992), 173-200. MR1146631 (92k:35241)

[14] K. Deng and M. Xu, On solutions of a singular diffusion equation, Nonlinear Anal. 41 (2000), 489-500. MR 1762159 (2001d:35102)

[15] J. I. Diaz, G. Hetzer, and L. Tello, An energy balance climate model with hysteresis, Nonlinear Anal. 64 (2006), 2053-2074. MR2211199 (2007a:35076)

[16] M. Fabrizio and A. Morro, A boundary condition with memory in electromagnetism, Arch. Rational Mech. Anal. 136 (1996), 359-381. MR:1423012 (97k:78012)

[17] A. Friedman, Partial differential equations of parabolic type, Prentice-Hall Inc., Englewood Cliffs, NJ, 1964. MR0181836(31:6062)

[18] M. Grasselli and V. Pata, A reaction-diffusion equation with memory, Discrete Contin. Dyn. Syst. Ser. A 15 (2006), 1079-1088. MR2224498 (2006m:35184)

[19] G. Hetzer, A quasilinear functional reaction-diffusion equation from climate modeling, Nonlinear Anal. 30 (1997), 2547-2556. MR.1602947 (99d:35083)

[20] D. Hirata, Blow-up for a class of semilinear integro-differential equations of parabolic type, Math. Methods Appl. Sci. 22 (1999), 1087-1100. MR.1706106 (2000e:35111)

[21] B. Hu, Remarks on the blowup estimates for solution of the heat equation with a nonlinear boundary condition, Differential Integral Equations 9 (1996), 891-901. MR.1392086 (97e:35092)

[22] B. Hu and H. M. Yin, Critical exponents for a system of heat equations coupled in a non-linear boundary condition, Math. Methods Appl. Sci. 19 (1996), 1099-1120. MR.1409541 (97h:35115) 
[23] B. Hu and H. M. Yin, The profile near blowup time for solutions of the heat equations with a nonlinear boundary condition, Trans. Amer. Math. Soc. 346 (1994), 117-135. MR1270664 (95c:35040)

[24] W. E. Kastenberg, Space dependent reactor kinetics with positive feedback, Nukleonki 11 (1968), 126-130.

[25] A. I. Kozhanov, Parabolic equations with nonlocal nonlinear source, Siberian Math. J. 35 (1994), 945-956. MR.1308236 (95k:35100)

[26] O. A. Ladyzhenskaja, V. A. Solonnikov, and N. N. Ural'ceva, Linear and quasilinear equations of parabolic type, Amer. Math. Soc., Providence, RI, 1967. MR0241822 (39:3159b)

[27] H. A. Levine, S. Pamuk, B. Sleeman, and M. Nilsen-Hamilton, Mathematical modeling of capillary formation and development in tumor angiogenesis: penetration into the stroma, Bull. Math. Biol. 63 (2001), 801-863.

[28] J. Lopez Gomez, V. Marquez, and N. Wolanski, Blow up results and localization of blow up points for the heat equation with a nonlinear boundary condition, J. Differential Equations 92 (1991), 384-401. MR 1120912 (92j:35098)

[29] Y. Li and C. Xie, Blow-up for semilinear parabolic equations with nonlinear memory, Z. Angew. Math. Phys. 55 (2004), 15-27. MR2033857 (2004m:35136)

[30] L. T. P. Ngoc, L. N. K. Hang, and N. T. Long, On a nonlinear wave equation associated with the boundary conditions involving convolution, Nonlinear Anal. 70 (2009), 3943-3965. MR2515312

[31] W. E. Olmstead, S. H. Davis, S. Rosenblat, and W. L. Kath, Bifurcation with memory, SIAM J. Appl. Math. 46 (1986), 171-188. MR833472 (87f:35020)

[32] C. V. Pao, Solution of a nonlinear integrodifferential system arising in nuclear reactor dynamics, J. Math. Anal. Appl. 48 (1974), 470-492. MR0356795 (50:9264)

[33] G. Propst and J. Prüss, On wave equations with boundary dissipation of memory type, J. Integral Equations Appl. 8 (1996), 99-123. MR1391147 (97d:35122)

[34] D. F. Rial and J. D. Rossi, Blow-up results and localization of blow-up points in an $\mathrm{N}$-dimensional smooth domain, Duke Math. J. 88 (1997), 391-405. MR.1455526 (98i:35079)

[35] P. Souplet, Monotonicity of solutions and blow-up for semilinear parabolic equations with nonlinear memory, Z. Angew. Math. Phys. 55 (2004), 28-31. MR2033858 (2004k:35199)

[36] P. Souplet, Blow-up in nonlocal reaction-diffusion equations, SIAM J. Math. Anal. 29 (1998), 13011334. MR 1638054 (99h:35104)

[37] P. Souplet, Nonexistence of global solutions to some differential inequalities of the second order and applications, Portugal. Math. 52 (1995), 289-299. MR.1355469 (96g:35220)

[38] P. Vernole, A time dependent parabolic initial boundary value delay problem, J. Integral Equations Appl. 6 (1994), 427-444. MR1312525 (95m:34147)

[39] Y. Yamada, Asymptotic stability for some systems of semilinear Volterra diffusion equations, J. Differential Equations 52 (1984), 295-326. MR744299 (85h:45005)

[40] Y. Yamada, On a certain class of semilinear Volterra diffusion equations, J. Math. Anal. Appl. 88 (1982), 433-451. MR667070 (84b:92062)

[41] J. Yong and X. Zhang, Heat equations with memory, Nonlinear Anal. 63 (2005), e99-e108. 